\title{
VARIANT OF THE LANCASTER DIPLOPIA TEST*
}

\author{
BY \\ JOHN FOSTER \\ With the assistance of E. C. Pemberton and Joan Bagshaw
}

Leeds General Infirmary

THE Lancaster diplopia test (Lancaster, 1939) is simple and sensitive, shows topical cyclophoria easily, and is applicable to alternators with abnormal retinal correspondence, so that in these respects it must be regarded as superior to the Hess test (Hess, 1916). The Lancaster method of recording, however, is less lucid, and attempts have been made, for example by Tulloch (1950) and Hugonnier (1952), to combine the two tests. Tulloch projected light from a green Lancaster torch on to a Hess background studded with red lights, reversing the red and green goggles to record secondary deviation.

After further experiments to improve the method of recording, we have devised an even simpler background consisting of a grey Essex board, $39^{\prime \prime} \times 39^{\prime \prime}$, on which is marked in black a central spot surrounded by a black square at $11^{\prime \prime}$ distance (i.e. with the centres of each side of the square at $5 \frac{1}{2}$ " from the spot, representing a $15^{\circ}$ excursion of the eye) and another at $28^{\prime \prime}$ (equivalent approximately to $30^{\circ}$ ). The Lancaster test with two torches is so sensitive that one can eliminate the small squares of the Hess chart without loss of " significant form".

The patient is placed in semi-darkness at half a metre from the centre of the board with his chin on a rest. A streak of white light (to avoid confusion with the coloured lights used later) is then swept across the board, and the edge of the area in which it appears to be double is marked by white pins.

The patient then puts on the red-green goggles with the red glass over the right eye, and the examiner projects a streak of light with a red torch on to the four corners of either the inner or the outer square (as suggested by the area of diplopia) and the central spot. The patient holding the green torch attempts to place the streak of light from it so as to cover or form a cross with the red streak.

T-shaped hat-pins dipped in green enamel are stuck in the board to correspond with position and rotation of the streak of light projected by the patient's torch. This plots the position of the left eye under conditions of dissociation.

The examiner and patient exchange torches, the process is repeated, and the secondary (or primary) deviation is recorded on the board by similar red pins. The board now carries a line of white pins to indicate the edge of the area of diplopia, and five green and five red pins (Fig. 1, opposite).

The two squares and the central spot are rubber stamped to scale on the patient's notes and the position of the pins is transferred to this diagram, which is

* Received for publication January 1, 1953. 
$5 \mathrm{~cm}$. square. We have found it possible to do this by estimate without material loss of accuracy. The area of diplopia is hatched in, the positions of the red pins are indicated by a red pencil, and those of the green pins by a green pencil. The points are joined by a line, as in the Hess test, but the primary and secondary deviations are recorded on the same diagram.

Cyclophoria if marked or unusual is recorded by straight coloured lines, which are joined by a fainter line.

Where the test is a second or subsequent one and the condition is stationary, transcription may be avoided

FIG. 1.-Method of testing.-The round pins mark the edge of the zone of diplopia, the oblong pins mark the position of the streaks of light projected by the patient's and the examiner's torches. Owing to the expense of colour printing, they are here shown as white. Although the test is conducted in semi-darkness, the board is momentarily illuminated by the photographer's flashlight. The markings of earlier experiments are still faintly visible on the board as well as the two squares recommended. by comparing the board with earlier records.

\section{Advantages}

Time Factor. - The time required to explain and to record the test on paper by this method is the same as that for the conventional Hess test. The time taken in plotting (including the field of diplopia) is about 35 seconds longer per case. To speed this up we are experimenting with a board of grey painted sheet iron, the position of the streaks of light being indicated by small magnets* which are enamelled red, green, or white, to correspond with the pins previously described. This appears to be a little more rapid in use than the wood or composition board with inserted pins, but the use of the more complex magnet board is probably only justified in a large clinic where the instrument is much used.

Cost.-The test is inexpensive. The Essex composition board backed by plywood is home-made; the three Aimark torches, the goggles, pins, and rubber stamps cost together about $\mathfrak{£} 7$.

Interpretation.-The representation of primary and secondary deviation with the field of diplopia on a single diagram, gives, we feel, a much clearer grasp of the initial position and subsequent progress of the case than two Hess charts and a separate perimetric diplopia record. It may be objected that the area tested for diplopia is relatively small and that the squares are not accurate in spherical projection, but the error from the latter is only slight, and patients whose diplopia is outside this area do not, as a rule, complain much of it.

\footnotetext{
* M.1418A $0.75^{\prime \prime} \times 0.2^{\prime \prime} \times 0.7^{\prime \prime}$ of 35 per cent. cobalt steel, made by James Neill and Co., of Sheffield.
} 
Application

Fig. 2 is an example of a record by our method of a patient with paresis of the right superior oblique. Two months later the patient claimed considerable diminution of symptoms. The test shows that the vertical deviation and total area of diplopia are undiminished, but that relaxation of the left inferior rectus and increased contracture of the right inferior oblique have displaced the centre of gravity of the diplopia from the lower field (Fig. 3).

Alternating Squint.-An interesting application of the method is to longstanding paretic squints complicated by alternating suppression, for which the Hess test is useless. The Lancaster test will record these squints, but, if abnormal retinal correspondence is present, it records the false or subjective angle. Fig. 4 is the record of a patient with a paresis of the left superior oblique since the age of 3 , who developed difficulty with close work in adult life. The objective angle (by synoptophore) in the primary position was $+10^{\circ}$ $\mathrm{L} / \mathrm{R}$ 39. The subjective angle ccrresponded to Fig. 4, i.e., abnormal retinal correspondence is recorded in both horizontal and vertical planes.

Cyclophoria.-As indicated by Maddox (1898), pre-existing cyclophoria may confuse the diagnosis of the paralysed muscle. Nevertheless, so striking is the reversal of the cyclophoria seen in different parts of the field in certain cases (Fig. 5) and so difficult is it to explain that we wonder if conventional beliefs on this subject are well founded. Nevertheless, the degree of cyclophoria exhibited is of service in deciding whether prismatic correction will be helpful to the patient, and the Lancaster test may prove a method of analysis better capable of settling disputed points than its predecessors.

Ophthalmoplegia and Exophthalmos.-Rundle and Wilson (1944) have recorded limitation of the field of fixation in this condition. Certain intelligent patients with thyrotrophic exophthalmos stated in the course of a Lancaster diplopia test that they were "no longer looking directly at the streak of light ". It is possible by making use of this observation to measure a limited field of fixation. It is best to record this separately, or the diagram becomes too crowded for clarity. It would appear that patients with exophthalmos are much more tolerant of diplopia than others whose deviation and area of diplopia are almost co-extensive, and we are investigating the possibility that limitation of movement may be in some way related to this for example, Fig. 6 is the record of a patient with an orbital tumour, who complained little of diplopia. Fig. 7 is the record of a patient with a similar degree of paralysis and a small central area of single binocular vision due to an intracranial aneurysm, who kept one eye constantly covered.

\section{REFERENCES}

Hess, W. R. (1916). Z. Augenheilk., 35, 201.

HUGONNIER, R. (1952). Bull. Soc. franç. Ophtal., 65, 271.

LanCaster, W. B. (1939). J. Amer. med. Ass., 113, 1413.

MADDOX, E. E. (1898). "Tests and Studies of the Ocular Muscles", p. 301. Wright, Bristol. Rundle, F. F., and Wilson, C. W. (1944). Clin. Sci., 5, 17.

Tulloch, A. K. (1950). Brit. orthoptic J., 7, 93. 


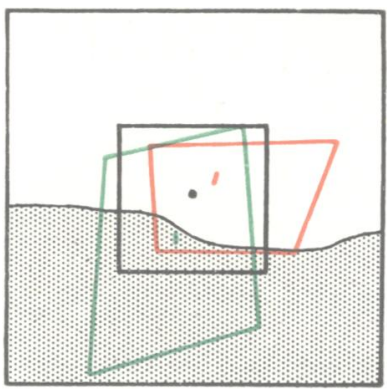

Fig. 2

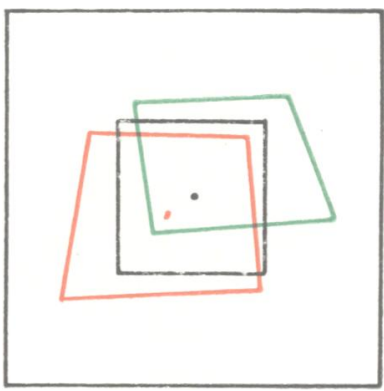

Fig. 4

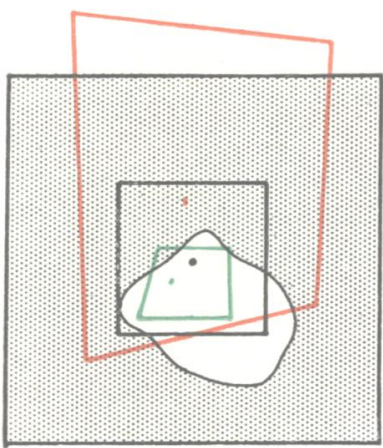

Fig. 6

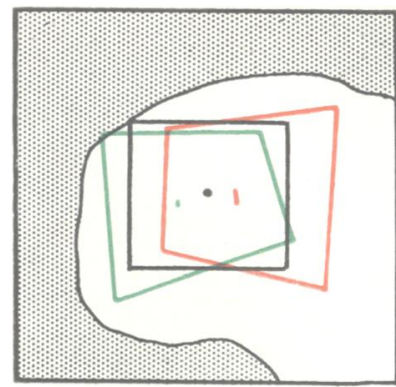

Fig. 3

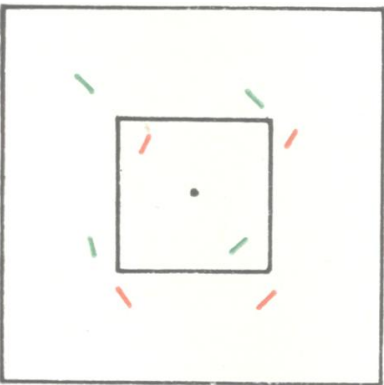

Fig. 5

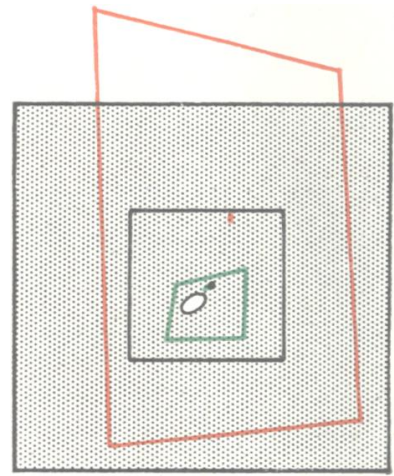

Fig. 7

FIG. 2.-Paresis of superior oblique.-The area in which the patient sees double is hatched. The position of the strip of light projected by the red torch when held by the patient is marked by the double line, and that of the green torch when held by the patient by the dotted line.

FIG. 3.-Elevation of diplopia.-Vertical deviation in the same patient is little altered, but as the area of diplopia is now more marked above than below, his symptoms are greatly diminished.

FIG. 4.-Abnormal correspondence.-This patient's squint started at the age of 3 and was recorded at the age of 21 . The objective angle on the synoptophore is $+10^{\circ} \mathrm{L} / \mathrm{R} 39$. The subjective angle corresponds with that recorded here. That is to say that the "false" angle in both horizontal and vertical planes is recorded by this method when abnormal retinal correspondence is present.

Fig. 5.-Cyclophoria.-The reversal of the cyclophoria in the upper and lower fields is difficult to explain on a conventional basis. Observational error was excluded by several repetitions.

FIG. 6.-Orbital tumour.-This patient has an orbital tumour with good vision in both eyes, but limitation of movement and gross paresis of the muscles of the left. The area of single binocular vision was apparently large enough for the patient, who complained little of diplopia.

FIG. 7.-Sub-arachnoid haemorrhage.-This patient has a similar, though slightly greater degree of paresis and a very small field of single binocular vision. She was completely incapacitated by diplopia and kept the eye permanently covered. 


\section{ANNOTATION}

MANY years ago I obtained a dozen pig's eyes for the purpose of trying different techniques in cataract extraction. Some of the eyes were left over, and my elder son, then aged about 10, wanted to know what they were for. I showed him how to remove a lens and he asked if he could try and succeeded at his first shot. Then he asked me, " Do you get a hundred guineas for doing that on a patient ?", to which I replied, "Occasionally". He thought that this would be a good way of earning a living but I thought it would be as well to let him see another side of our specialty. So a few weeks later on a wet Sunday, when the two boys having nothing better to do were being entertained by me in the consulting room, I made the elder work out his younger brother's refraction. It took some time to do and at the end I was asked, " Do you have to do this on every patient?", to which I replied that I had to, on practicaliy all. From that day forward ophthalmology as a future career became less attractive.

This experience brought home to me how deadly dull a whole day spent in refraction work could be, were it not for the endless variety in the characters of those who owned the eyes one was testing and in their responses to the tests one used. An interesting distinction between children and adults is shown in their replies to the familiar question, "Can you see the bottom line?" A child will nearly always say "Yes" or "No" as the case may be, whereas an adult prefers to read the letters aloud if he can see them. The child presumably expects to be believed or has the common sense not to bother himself to read out a long line of letters if he can avoid it, whereas the adult, having been in contact with the world for considerably longer, knows he must prove his statement.

Children's clear-cut logic can be a little upsetting at times. I remember once testing a small boy with the Maddox rod and explaining to him that he would see a red line and a light. I switched the light on and asked if he could see the red line, to which he replied with an uncompromising "No". I then covered the eye which was viewing the light and asked him if he could see the red line, but he still said he could not, so finally I asked him what he could see and he replied, " A red line". I told him that this was what I had been asking him about, and he replied "Oh no, you said I'd see a red lion".

The story of the small boy who refused to read the letters because they did not spell anything is too well known to need recital in detail, but occasionally the same thing happens in adults, only in their case the refusal is because the letters, not being clearly seen, appear to spell a word which from motives of decency they refuse to pronounce. One such line occurs as $6 / 12$ in a well-known set of test types.

Annotations in the past have usually carried a moral, but it is difficult to find one here unless it be that even in refraction we can at times "Behold the bright original appear".

\section{CORRIGENI)A}

The caption to Fig. 2 in the article entitled "Variant of the Lancaster Diplopia Test " by John Foster which appeared in the British Journal of Ophthalmology, 1953, 37, 378, should read " The position of the strip of light projected by the red torch when held by the patient is marked by the red line, and that of the green torch when held by the patient by the green line".

The antibiotic "Aerosporin" (see the footnote to an article entitled "Ps. Pyocyaneus Eye Infection" by Marguerite Macdonald, British Journal of Ophthalmology, 1953, 37, 371) is manufactured by Messrs Burroughs Wellcome and Co., England. 